\title{
Optimality conditions in optimal control problems for a Gursa-Darboux system with a multipoint objective functional
}

\author{
Sh.Sh. Suleymanova
}

Institute of Control Systems of Azerbaijan National Academy of Sciences, Baku, Azerbaijan

\begin{tabular}{|c|c|}
\hline A R T I C L E I N F O & A B S T RA C T \\
\hline $\begin{array}{l}\text { Article history: } \\
\text { Received 12.07.2021 } \\
\text { Received in revised form } 30.07 .2021 \\
\text { Accepted 10.08.2021 } \\
\text { Available online } 29.12 .2021\end{array}$ & $\begin{array}{l}\text { We consider one linear optimal control problem described by a } \\
\text { system of hyperbolic equations with the Goursat boundary condi- } \\
\text { tion and a multipoint linear objective functional. A necessary and } \\
\text { sufficient optimality condition of the Pontryagin maximum princi- } \\
\text { ple type is proved. }\end{array}$ \\
\hline $\begin{array}{l}\text { Keywords: } \\
\text { Permissible control } \\
\text { Maximum principle } \\
\text { Optimality condition } \\
\text { Multipoint functional }\end{array}$ & \\
\hline
\end{tabular}

\section{Introduction}

There are many studies devoted to various optimal control problems for Goursat-Darboux systems (see, e.g., [1-8]), in which a number of necessary optimality conditions are established under various assumptions.

Beginning with [9-13], optimal control problems described in different time intervals by various ordinary differential equations were studied. Such optimal control problems are called compound optimal control problems or variable-structure optimal control problems.

In this article, one linear optimal control problem is considered, described in different domains by two systems of hyperbolic equations with a multipoint quality functional. A necessary and sufficient optimality condition is proved. The case of a nonlinear convex differentiable objective functional is also investigated. A sufficient optimality condition of the Pontryagin type is proved.

\section{Problem statement}

Suppose that we have the specified rectangles $D_{1}=\left[t_{0}, t_{1}\right] \times\left[x_{0}, x_{1}\right], D_{2}=\left[t_{1}, t_{2}\right] \times$ $\left[x_{0}, x_{1}\right],\left(t_{0}<t_{1}<t_{2}, x_{0}<x_{1}\right),\left(T_{i}, X_{i}\right), i=\overline{1, k}\left(t_{0}<T_{1}<T_{2}<\cdots<T_{k} \leq t_{1} ; x_{0}<X_{1}<X_{2}<\right.$ $\left.\cdots<X_{k} \leq x_{1}\right), \theta_{i}, i=\overline{1, k}\left(t_{1}<\theta_{1}<\theta_{2}<\cdots<\theta_{k} \leq t_{2}\right)$ are specified points; $U_{1} \subset R^{r}, U_{2} \subset R^{q}$ are specified non-empty and bounded sets; $u_{i}(t, x), i=1,2$ are measurable and bounded respectively, $r$ - and $q$-dimensional vector functions satisfying the constraints

$$
\begin{aligned}
& u_{1}(t, x) \in U_{1} \subset R^{r},(t, x) \in D_{1}, \\
& u_{2}(t, x) \in U_{2} \subset R^{q},(t, x) \in D_{2} .
\end{aligned}
$$

E-mail address: kmansimov@mail.com (Sh.Sh. Suleymanova). 
The pair $\left(u_{1}(t, x), u_{2}(t, x)\right)$ with the above properties will be called admissible control.

Assume that the controlled process is described by systems of linear hyperbolic equations with boundary conditions of the Goursat type, i.e.,

$$
\begin{gathered}
\frac{\partial^{2} z_{1}}{\partial t \partial x}=A_{1}(t, x) z_{1}+B_{1}(t, x) \frac{\partial z_{1}}{\partial t}+C_{1}(t, x) \frac{\partial z_{1}}{\partial x}+f_{1}\left(t, x, u_{1}\right), \\
z_{1}\left(t_{0}, x\right)=a(x), x \in\left[x_{0}, x_{1}\right], \\
z_{1}\left(t, x_{0}\right)=b_{1}(t), t \in\left[t_{0}, t_{1}\right], \\
a\left(x_{0}\right)=b_{1}\left(t_{0}\right), \\
\frac{\partial^{2} z_{2}}{\partial t \partial x}=A_{2}(t, x) z_{2}+B_{2}(t, x) \frac{\partial z_{2}}{\partial t}+C_{2}(t, x) \frac{\partial z_{2}}{\partial x}+f_{2}\left(t, x, u_{2}\right), \\
z_{2}\left(t_{1}, x\right)=B(x) z_{1}\left(t_{1}, x\right), x \in\left[x_{0}, x_{1}\right], \\
z_{2}\left(t, x_{0}\right)=b_{2}(t), t \in\left[t_{1}, t_{2}\right], \\
B\left(x_{0}\right) z_{1}\left(t_{1}, x_{0}\right)=b_{2}\left(t_{1}\right) .
\end{gathered}
$$

Here, $A_{i}(t, x), B_{i}(t, x), C_{i}(t, x) i=1,2$ are specified $(n \times n)$ measurable bounded matrix functions; $f_{i}\left(t, x, u_{i}\right), i=1,2$ are specified $n$-dimensional vector functions continuous in the set of variables; $a(x), b_{i}(t), i=1,2$ are specified absolutely continuous vector functions; $B(x)$ is a specified continuously differentiable $(n \times n)$ matrix functions.

It is assumed that for a specified admissible control $\left(u_{1}(t, x), u_{2}(t, x)\right)$, problem (2)-(5) has a unique absolutely continuous solution $\left(z_{1}(t, x), z_{2}(t, x)\right)$ (see, e.g., [3]).

Suppose that $c_{i}, d_{i}$ are specified $n$-dimensional vectors.

Let us consider the problem of finding the minimum value of the multipoint functional

$$
S\left(u_{1}, u_{2}\right)=\sum_{i=1}^{k} c_{i}{ }^{\prime} z_{1}\left(T_{i}, X_{i}\right)+\sum_{i=1}^{k} d_{i}{ }^{\prime} z_{2}\left(\theta_{i}, X_{i}\right)
$$

under constraints (1)-(5).

The admissible control $\left(u_{1}(t, x), u_{2}(t, x)\right)$ satisfying the minimum value to functional (6) under constraints (1)-(5) will be called an optimal control, and the corresponding process $\left(u_{1}(t, x), u_{2}(t, x), z_{1}(t, x), z_{2}(t, x)\right)-$ an optimal process.

\section{Formula for the increment of the objective functional and the optimality condition}

Assuming that $\left(u_{1}(t, x), u_{2}(t, x), z_{1}(t, x), z_{2}(t, x)\right)$ a fixed admissible process, we denote by $\left(\bar{u}_{1}(t, x)=u_{1}(t, x)+\Delta u_{1}(t, x), \bar{u}_{2}(t, x)=u_{2}(t, x)+u_{2}(t, x), \bar{z}_{1}(t, x)=z_{1}(t, x)+\right.$ $\left.\Delta z_{1}(t, x), \overline{z_{2}}(t, x)=z_{2}(t, x)+\Delta z_{2}(t, x)\right)$ the arbitrary admissible process and write down the increment of functional (6).

$$
\Delta S\left(u_{1}, u_{2}\right)=S\left(\bar{u}_{1}, \bar{u}_{2}\right)-S\left(u_{1}, u_{2}\right)=\sum_{i=1}^{k} c_{i}{ }^{\prime} \Delta z_{1}\left(T_{i}, X_{i}\right)+\sum_{i=1}^{k} d_{i}{ }^{\prime} \Delta z_{2}\left(\theta_{i}, X_{i}\right) .
$$

It is clear that the increment $\left(\Delta z_{1}(t, x), \Delta z_{2}(t, x)\right)$ of the state $\left(z_{1}(t, x), z_{2}(t, x)\right)$ is the solution to the boundary value problem

$$
\begin{aligned}
& \frac{\partial^{2} \Delta z_{1}(t, x)}{\partial t \partial x}=A_{1}(t, x) \Delta z_{1}(t, x)+B_{1}(t, x) \frac{\partial \Delta z_{1}(t, x)}{\partial t}+ \\
& +C_{1}(t, x) \frac{\partial \Delta z_{1}(t, x)}{\partial x}+f_{1}\left(t, x, \bar{u}_{1}(t, x)\right)-f_{1}\left(t, x, u_{1}(t, x)\right)
\end{aligned}
$$




$$
\begin{gathered}
z_{1}\left(t_{0}, x\right)=0, x \in\left[x_{0}, x_{1}\right], \\
z_{1}\left(t, x_{0}\right)=0, t \in\left[t_{0}, t_{1}\right], \\
\frac{\partial^{2} \Delta z_{2}(t, x)}{\partial t \partial x}=A_{2}(t, x) \Delta z_{2}(t, x)+B_{2}(t, x) \frac{\partial \Delta z_{2}(t, x)}{\partial t}+ \\
+C_{2}(t, x) \frac{\partial \Delta z_{2}(t, x)}{\partial x}+f_{2}\left(t, x, \bar{u}_{2}(t, x)\right)-f_{2}\left(t, x, u_{2}(t, x)\right), \\
z_{2}\left(t_{1}, x\right)=B(x) \Delta z_{1}\left(t_{1}, x\right), x \in\left[x_{0}, x_{1}\right], \\
z_{2}\left(t, x_{0}\right)=0, t \in\left[t_{1}, t_{2}\right] .
\end{gathered}
$$

Assuming that $\psi_{i}(t, x), i=1,2$ are as yet unknown $n$-dimensional vector functions, we introduce the notation

$$
\begin{gathered}
\Delta_{\bar{u}_{i}} f_{i}[t, x] \equiv f_{i}\left(t, x, \bar{u}_{i}(t, x)\right)-f_{i}\left(t, x, u_{i}(t, x)\right), \\
H\left(t, x, u_{i}, \psi_{i}\right)=\psi_{i}^{\prime} f_{i}\left(t, x, u_{i}\right), \\
\Delta_{\bar{u}_{i}} H\left[t, x, \psi_{i}\right] \equiv H\left(t, x, \bar{u}_{\imath}, \psi_{i}\right)-H\left(t, x, u_{i}, \psi_{i}\right) .
\end{gathered}
$$

From relations (8), (10) we obtain that

$$
\begin{gathered}
\int_{t_{i-1}}^{t_{i}} \int_{x_{0}}^{x_{1}} \psi_{i}^{\prime}(t, x) \frac{\partial^{2} \Delta z_{i}(t, x)}{\partial t \partial x} d t d x= \\
=\int_{t_{i-1}}^{t_{i}} \int_{x_{0}}^{x_{1}}\left[\psi _ { i } ^ { \prime } ( t , x ) \left(A_{i}(t, x) \Delta z_{i}(t, x)+B_{i}(t, x) \frac{\partial \Delta z_{i}(t, x)}{\partial t}+\right.\right. \\
\left.\left.+C_{i}(t, x) \frac{\partial \Delta z_{i}(t, x)}{\partial x}\right)+\Delta_{\bar{u}_{i}} H\left[t, x, \psi_{i}\right]\right] d t d x, i=\overline{1,2 .}
\end{gathered}
$$

Taking into account boundary conditions (9), (11), we obtain that

$$
\begin{gathered}
\Delta z_{1}(t, x)=\int_{t_{0}}^{t} \int_{x_{0}}^{x} \frac{\partial^{2} \Delta z_{1}(\tau, s)}{\partial \tau \partial s} d \tau d s, \\
\frac{\partial \Delta z_{1}(t, x)}{\partial t}=\int_{x_{0}}^{x} \frac{\partial^{2} \Delta z_{1}(t, s)}{\partial t \partial s} d s, \\
\frac{\partial \Delta z_{1}(t, x)}{\partial x}=\int_{t_{0}}^{t} \frac{\partial^{2} \Delta z_{1}(\tau, x)}{\partial \tau \partial x} d \tau, \\
\left.\frac{\partial \Delta z_{2}(t, x)}{\partial t}=\int_{x_{0}}^{t} \frac{\partial^{2} \Delta z_{2}(t, s)}{\partial t \partial s} d s, x\right) \\
\frac{B(x) \Delta z_{1}\left(t_{1}, x\right)+\int_{t_{1}}^{x} \frac{\partial^{2} \Delta z_{2}(\tau, s)}{\partial \tau \partial s} d \tau d s,}{\partial x}=\frac{\partial B(x)}{\partial x} \Delta z_{1}\left(t_{1}, x\right)+B(x) \frac{\partial \Delta z_{1}\left(t_{1}, x\right)}{\partial x}+\int_{t_{1}}^{t} \frac{\partial^{2} \Delta z_{2}(\tau, x)}{\partial \tau \partial x} d \tau,
\end{gathered}
$$


Further, suppose that $\alpha_{i}(t, x), i=\overline{1, k}$ are characteristic functions of the rectangles $\left[t_{0}, T_{i}\right] \times$ $\left[x_{0}, X_{i}\right], \beta_{i}(t, x) ; i=\overline{1, k}$ are characteristic functions of the rectangles $\left[t_{1}, \theta_{i}\right] \times\left[x_{0}, X_{i}\right]$, and $\gamma_{i}(x), i=\overline{1, k}$ are characteristic functions of the segments $\left[x_{0}, X_{i}\right], i=\overline{1, k}$.

Taking into account identities (13) and (16), we obtain that

$$
\begin{gathered}
\Delta z_{1}\left(T_{i}, X_{i}\right)=\int_{t_{0}}^{t_{1}} \int_{x_{0}}^{x_{1}} \alpha_{i}(t, x) \frac{\partial^{2} \Delta z_{1}(t, x)}{\partial t \partial x} d x d t \\
\Delta z_{2}\left(\theta_{i}, X_{i}\right)=B\left(X_{i}\right) \Delta z_{1}\left(t_{1}, X_{i}\right)+\int_{t_{1}}^{\theta_{i}} \int_{x_{0}}^{X_{i}} \frac{\partial^{2} \Delta z_{2}(t, x)}{\partial t \partial x} d x d t= \\
=\int_{t_{1}}^{t_{2}} \int_{x_{0}}^{x_{1}} B\left(X_{i}\right) \gamma_{i}(x) \frac{\partial^{2} \Delta z_{1}(t, x)}{\partial t \partial x} d x d t+\int_{t_{1}}^{t_{2}} \int_{x_{0}}^{x_{1}} \beta_{i}(t, x) \frac{\partial^{2} \Delta z_{1}(t, x)}{\partial t \partial x} d x d t .
\end{gathered}
$$
resented as

Given identities (13)-(20), the formula for increment (7) of objective functional (6) can be rep-

$$
\begin{aligned}
& \Delta S\left(u_{1}, u_{2}\right)=\int_{t_{0}}^{t_{1}} \int_{x_{0}}^{x_{1}} \sum_{i=1}^{k} c_{i}^{\prime} \alpha_{i}(t, x) \frac{\partial^{2} \Delta z_{1}(t, x)}{\partial t \partial x} d x d t+ \\
& +\int_{t_{0}}^{t_{1}} \int_{x_{0}}^{x_{1}} \sum_{i=1}^{k} d_{i}{ }^{\prime} B\left(X_{i}\right) \gamma_{i}(x) \frac{\partial^{2} \Delta z_{1}(t, x)}{\partial t \partial x} d x d t+\int_{t_{1}}^{t_{2}} \int_{x_{0}}^{x_{1}} \sum_{i=1}^{k} \beta_{i}(t, x) d_{i}{ }^{\prime} \frac{\partial^{2} \Delta z_{2}(t, x)}{\partial t \partial x} d x d t+ \\
& +\int_{t_{0}}^{t_{1}} \int_{x_{0}}^{x_{1}} \psi_{1}^{\prime}(t, x) \frac{\partial^{2} \Delta z_{1}(t, x)}{\partial t \partial x} d x d t-\int_{t_{0}}^{t_{1}} \int_{x_{0}}^{x_{1}}\left[\int_{t}^{t_{1}} \int_{x}^{x_{1}} \psi_{1}^{\prime}(\tau, s) A_{1}(\tau, s) d s d \tau\right] \frac{\partial^{2} \Delta z_{1}(t, x)}{\partial t \partial x} d x d t- \\
& -\int_{t_{0}}^{t_{1}} \int_{x_{0}}^{x_{1}}\left[\int_{x}^{x_{1}} \psi_{1}^{\prime}(t, s) B_{1}(t, s) d s\right] \frac{\partial^{2} \Delta z_{1}(t, x)}{\partial t \partial x} d x d t- \\
& -\int_{t_{0}}^{t_{1}} \int_{x_{0}}^{x_{1}}\left[\int_{t}^{t_{1}} \psi_{1}^{\prime}(\tau, x) C_{1}(\tau, x) d \tau\right] \frac{\partial^{2} \Delta z_{1}(t, x)}{\partial t \partial x} d x d t+\int_{t_{2}}^{t_{2}} \int_{x_{0}}^{x_{1}} \psi_{2}^{\prime}(t, x) \frac{\partial^{2} \Delta z_{2}(t, x)}{\partial t \partial x} d x d t- \\
& -\int_{t_{1}}^{t_{2}} \int_{x_{0}}^{x_{1}}\left[\int_{t_{2}}^{t_{2}} \int_{t_{1}}^{t_{2}} \psi_{2}^{\prime}(\tau, s) C_{2}(\tau, s) B(s) d s d \tau\right] \frac{\partial^{2} \Delta z_{2}(t, x)}{\partial t \partial x} d x d t- \\
& -\int_{t_{1}}^{t_{2}} \int_{x_{0}}^{x_{1}}\left[\int_{x}^{x_{1}} \psi_{2}^{\prime}(t, s) B_{2}(t, s) d s\right] \frac{\partial^{2} \Delta z_{2}(t, x)}{\partial t \partial x} d x d t- \\
& -\int_{t_{1}}^{t_{2}} \int_{t_{1}}^{x_{1}}\left[\int_{t}^{x_{1}} \psi_{2}^{\prime}(\tau, x) C_{2}(\tau, x) d \tau\right] \frac{\partial^{2} \Delta z_{2}(t, x)}{\partial t \partial x} d x d t- \\
& -\int_{t_{0}}^{t_{1}} \int_{x_{0}}^{x_{1}} \Delta_{\bar{u}_{1}(t, x)} H\left[t, x, \psi_{1}\right] d x d t-\int_{t_{1}}^{t_{2}} \int_{x_{0}}^{x_{1}} \Delta_{\bar{u}_{2}(t, x)} H\left[t, x, \psi_{2}\right] d x d t .
\end{aligned}
$$

If we assume that $\psi_{i}(t, x), i=1,2$ satisfy the relations 


$$
\begin{aligned}
& \psi_{1}(t, x)=-\sum_{i=1}^{k} c_{i} d_{i}(t, x)-\sum_{i=1}^{k} \gamma_{i}(x) B^{\prime}\left(X_{i}\right) d_{i}+\int_{t}^{t_{1}} \int_{x}^{x_{1}} \psi_{1}^{\prime}(\tau, s) A_{1}(\tau, s) d s d \tau+ \\
&+ \int_{x}^{x_{1}} \psi_{1}^{\prime}(t, s) B_{1}(t, s) d s+\int_{t}^{t_{2}} \psi_{1}^{\prime}(\tau, x) C_{1}(\tau, x) d \tau+\int_{t_{1}}^{x_{1}} \int_{x}^{t_{2}} B^{\prime}(s) A_{2}(\tau, s) d s d \tau+ \\
&+\int_{t_{1}}^{t_{2}} B^{\prime}(x) C_{2}(\tau, x) \psi_{2}(\tau, x) d \tau+\int_{t_{1}}^{x_{1}} \frac{B^{\prime}(s)}{\partial s} C_{2}(\tau, s) \psi_{2}(\tau, s) d s d \tau, \\
& \psi_{2}(t, x)=-\sum_{i=1}^{k} \beta_{i}(t, x) d_{i}+\int_{t}^{t_{2}} \int_{x}^{x_{1}} A_{2}(\tau, s) \psi_{1}(\tau, s) d s d \tau+\int_{x}^{t_{2}} \psi_{2}^{\prime}(t, s) B_{2}(t, s) d s \\
&+\int_{t} \psi_{2}^{\prime}(\tau, x) C_{2}(\tau, x) d \tau .
\end{aligned}
$$

then the formula for increment (21) takes the form

$$
\Delta S\left(u_{1}, u_{2}\right)=-\int_{t_{0}}^{t_{1}} \int_{x_{0}}^{x_{1}} \Delta_{\bar{u}_{1}(t, x)} H\left[t, x, \psi_{1}\right] d x d t-\int_{t_{1}}^{t_{2}} \int_{x_{0}}^{x_{1}} \Delta_{\bar{u}_{2}(t, x)} H\left[t, x, \psi_{2}\right] d x d t .
$$

As we can see, relations (22), (23) represent a system of linear Volterra integral equations with respect to $\psi_{1}(t, x), \psi_{2}(t, x)$, respectively. Using formula (24), we prove

Theorem 1. Under the assumptions made, for the optimality of the admissible control $\left(u_{1}(t, x), u_{2}(t, x)\right)$ it is necessary and sufficient that the inequalities

$$
\begin{aligned}
& \int_{t_{0}}^{t_{1}} \int_{x_{0}}^{x_{1}} \Delta_{\bar{u}_{1}(t, x)} H\left[t, x, \psi_{1}\right] d x d t \leq 0, \\
& \int_{t_{1}}^{t_{2}} \int_{x_{0}}^{x_{1}} \Delta_{\bar{u}_{2}(t, x)} H\left[t, x, \psi_{2}\right] d x d t \leq 0
\end{aligned}
$$

hold for all $v_{i}(t, x) \in U_{i},(t, x) \in D_{i}, i=1,2$.

Proof. Necessity. Suppose that $\left(u_{1}(t, x), u_{2}(t, x)\right)$ is an optimal control. Then we have that

$$
\begin{gathered}
S\left(v_{1}(t, x), u_{2}(t, x)\right)-S\left(u_{1}(t, x), u_{2}(t, x)\right)= \\
=-\int_{t_{0}}^{t_{1}} \int_{x_{0}}^{x_{1}} \Delta_{v_{1}(t, x)} H\left[t, x, \psi_{1}\right] d x d t \geq 0, \\
S\left(u_{1}(t, x), v_{2}(t, x)\right)-S\left(u_{1}(t, x), u_{2}(t, x)\right)= \\
=-\int_{t_{1}}^{t_{2}} \int_{x_{0}}^{x_{1}} \Delta_{v_{2}(t, x)} H\left[t, x, \psi_{1}\right] d x d t \geq 0 .
\end{gathered}
$$

The last inequalities imply the correctness of relations (25), (26).

Sufficiency. Suppose that relations (25), (26) hold. Then it follows from increment formula (24) that

$$
S\left(v_{1}, v_{2}\right)-S\left(u_{1}, u_{2}\right)=
$$




$$
=-\int_{t_{0}}^{t_{1}} \int_{x_{0}}^{x_{1}} \Delta_{v_{1}(t, x)} H\left[t, x, \psi_{1}\right] d x d t-\int_{t_{1}}^{t_{2}} \int_{x_{0}}^{x_{1}} \Delta_{v_{2}(t, x)} H\left[t, x, \psi_{1}\right] d x d t \geq 0 .
$$

This proves the sufficiency, since by virtue of (25), (26)

$$
S\left(u_{1}, u_{2}\right) \leq S\left(v_{1}, v_{2}\right)
$$

for arbitrary $\left(v_{1}(t, x), v_{2}(t, x)\right)$.

\section{The case of a nonlinear convex functional}

Suppose that it is required to find the minimum value of the functional

$$
S\left(u_{1}, u_{2}\right)=\varphi_{1}\left(z_{1}\left(T_{1}, X_{1}\right), \ldots, z_{1}\left(T_{k}, X_{k}\right)\right)+\varphi_{2}\left(z_{2}\left(\theta_{1}, X_{1}\right), \ldots, z_{2}\left(\theta_{k}, X_{k}\right)\right)
$$

under constraints (1)-(5).

Here, $\varphi_{1}\left(a_{1}, \ldots, a_{k}\right), \varphi_{2}\left(b_{1}, \ldots, b_{k}\right)$ are specified continuously differentiable, convex functions.

Using the Taylor formula, we write the increment of the quality functional corresponding to two admissible controls.

$$
\begin{gathered}
\Delta S\left(u_{1}, u_{2}\right)=\sum_{i=1}^{k} \frac{\partial \varphi_{i}{ }^{\prime}\left(z_{1}\left(T_{1}, X_{1}\right), \ldots, z_{1}\left(T_{k}, X_{k}\right)\right)}{\partial z_{1}} \Delta z_{1}\left(T_{i}, X_{i}\right)+ \\
+\sum_{i=1}^{k} \frac{\partial \varphi_{2}{ }^{\prime}\left(z_{2}\left(\theta_{1}, X_{1}\right), \ldots, z_{2}\left(\theta_{k}, X_{k}\right)\right)}{\partial z_{2}} \Delta z_{2}\left(\theta_{i}, X_{i}\right)+ \\
+o_{1}\left(\sum_{i=1}^{k}\left\|\Delta z_{1}\left(T_{i}, X_{i}\right)\right\|\right)+o_{2}\left(\sum_{i=1}^{k}\left\|\Delta z_{2}\left(\theta_{i}, X_{i}\right)\right\|\right) .
\end{gathered}
$$

Using identities (19), (20), we prove that

$$
\begin{gathered}
\sum_{i=1}^{k} \frac{\partial \varphi_{1}{ }^{\prime}\left(z_{1}\left(T_{1}, X_{1}\right), \ldots, z_{1}\left(T_{k}, X_{k}\right)\right)}{\partial z_{1}} \Delta z_{1}\left(T_{i}, X_{i}\right)= \\
=\int_{t_{0}} \int_{x_{0}}^{t_{1}} \sum_{i=1}^{x_{1}} \alpha_{i}(t, x) \frac{\partial \varphi_{1}{ }^{\prime}\left(z_{1}\left(T_{1}, X_{1}\right), \ldots, z_{1}\left(T_{k}, X_{k}\right)\right)}{\partial z_{1}} \frac{\partial^{2} \Delta z_{1}(t, x)}{\partial t \partial x} d x d t+ \\
+o_{1}\left(\sum_{i=1}^{k}\left\|\Delta z_{1}\left(T_{i}, X_{i}\right)\right\|\right), \\
=\int_{t_{1}}^{t_{2}} \int_{x_{0}}^{t_{1}} \sum_{i=1}^{t_{2}} \beta_{i}(t, x) \frac{\partial \varphi_{2}{ }^{\prime}\left(z_{2}\left(\theta_{1}, X_{1}\right), \ldots, z_{2}\left(\theta_{k}, X_{k}\right)\right)}{\partial z_{1}} \cdot \frac{\partial^{2} \Delta z_{2}(t, x)}{\partial t \partial x} d x d t+ \\
+\int_{t_{1}}^{x_{x_{0}}} \sum_{x_{0}}^{k} \sum_{i=1}^{k} \gamma_{i}(x) \frac{\partial \varphi_{2}{ }^{\prime}\left(z_{2}\left(\theta_{1}, X_{1}\right), \ldots, z_{2}\left(\theta_{k}, X_{k}\right)\right)}{\left.\left.\partial z_{2}, X_{1}\right), \ldots, z_{2}\left(\theta_{k}, X_{k}\right)\right)} \\
\partial\left(z_{i}\right) \frac{\partial^{2} \Delta z_{1}(t, x)}{\partial t \partial x} d x d t+
\end{gathered}
$$




$$
+o_{1}\left(\sum_{i=1}^{k}\left\|\Delta z_{2}\left(\theta_{i}, X_{i}\right)\right\|\right) .
$$
notation

Suppose $p_{i}(t, x), i=\overline{1,2}$ are as yet unknown $n$-dimensional vector functions. Introducing the

$$
\Delta_{\bar{u}_{i}} M\left[t, x, \psi_{i}\right] \equiv M\left(t, x, \bar{u}_{i}, p_{i}\right)-M\left(t, x, u_{i}, p_{i}\right), i=1,2
$$

and taking into account identities (13)-(18), (30), (31), the formula for increment (29) of functional (28) is written in the form

$$
\begin{aligned}
& \Delta S\left(u_{1}, u_{2}\right)=\int_{t_{0}}^{t_{1}} \int_{x_{0}}^{x_{1}} \sum_{i=1}^{k} \alpha_{i}(t, x) \frac{\partial \varphi_{1}{ }^{\prime}\left(z_{1}\left(T_{1}, X_{1}\right), \ldots, z_{1}\left(T_{k}, X_{k}\right)\right)}{\partial z_{1}} \cdot \frac{\partial^{2} \Delta z_{1}(t, x)}{\partial t \partial x} d x d t+ \\
& +\int_{t_{0}}^{t_{1}} \int_{x_{0}}^{x_{1}} \sum_{i=1}^{k} \gamma_{i}(x) \frac{\partial \varphi_{2}{ }^{\prime}\left(z_{2}\left(\theta_{1}, X_{1}\right), \ldots, z_{2}\left(\theta_{k}, X_{k}\right)\right)}{\partial z_{2}} \cdot B\left(X_{i}\right) \frac{\partial^{2} \Delta z_{1}(t, x)}{\partial t \partial x} d x d t+ \\
& +\int_{t_{1}}^{t_{2}} \int_{x_{0}}^{x_{1}} \sum_{i=1}^{k} \beta_{i}(t, x) \frac{\partial \varphi_{2}{ }^{\prime}\left(z_{2}\left(\theta_{1}, X_{1}\right), \ldots, z_{2}\left(\theta_{k}, X_{k}\right)\right)}{\partial z_{1}} \cdot \frac{\partial^{2} \Delta z_{2}(t, x)}{\partial t \partial x} d x d t+ \\
& +o_{1}\left(\sum_{i=1}^{k}\left\|\Delta z_{1}\left(T_{i}, X_{i}\right)\right\|\right)+o_{2}\left(\sum_{i=1}^{k}\left\|\Delta z_{2}\left(\theta_{i}, X_{i}\right)\right\|\right)+\int_{t_{0}}^{t_{1}} \int_{x_{0}}^{x_{1}} p_{1}{ }^{\prime}(t, x) \cdot \frac{\partial^{2} \Delta z_{1}(t, x)}{\partial t \partial x} d x d t- \\
& -\int_{t_{0}}^{t_{1}} \int_{x_{0}}^{x_{1}}\left[\int_{t}^{t_{1}} \int_{t_{1}}^{t_{1}} p_{x}^{x_{1}} p_{1}^{\prime}(\tau, s) A_{1}(\tau, s) d s d \tau\right] \frac{\partial^{2} \Delta z_{1}(t, x)}{\partial t \partial x} d x d t- \\
& -\int_{t_{0}}^{t_{1}} \int_{x_{0}}^{x_{1}}\left[\int_{x}^{x_{1}} p_{1}^{\prime}(t, s) B_{1}(t, s) d s\right] \frac{\partial^{2} \Delta z_{1}(t, x)}{\partial t \partial x} d x d t- \\
& -\int_{t_{0}}^{t_{1}} \int_{x_{0}}^{x_{1}}\left[\int_{t}^{t_{1}} p_{1}^{\prime}(\tau, x) C_{1}(\tau, x) d \tau\right] \frac{\partial^{2} \Delta z_{1}(t, x)}{\partial t \partial x} d x d t- \\
& -\int_{t_{0}}^{t_{1}} \int_{x_{0}}^{x_{1}} \Delta_{\bar{u}_{1}(t, x)} M\left[t, x, p_{1}\right] d x d t+\int_{t_{2}}^{t_{2}} \int_{x_{0}}^{x_{1}} p_{2}^{\prime}(t, x) \frac{\partial^{2} \Delta z_{2}(t, x)}{\partial t \partial x} d x d t- \\
& -\int_{t_{1}}^{t_{2}} \int_{x_{0}}^{x_{1}}\left[\int_{t}^{t_{2}} \int_{x}^{x_{1}} p_{2}^{\prime}(\tau, s) A_{2}(\tau, s) B(s) d s d \tau\right] \frac{\partial^{2} \Delta z_{1}(t, x)}{\partial t \partial x} d x d t- \\
& -\int_{t_{0}}^{t_{1}} \int_{x_{0}}^{x_{1}}\left[\int_{t_{1}}^{t_{2}} \int_{x}^{x_{1}} p_{2}^{\prime}(\tau, s) A_{2}(\tau, s) B(s) d s d \tau\right] \frac{\partial^{2} \Delta z_{2}(t, x)}{\partial t \partial x} d x d t- \\
& -\int_{t_{1}}^{t_{2}} \int_{x_{0}}^{x_{1}}\left[\int_{x}^{x_{1}} p_{2}^{\prime}(t, s) B_{2}(t, s) d s\right] \frac{\partial^{2} \Delta z_{2}(t, x)}{\partial t \partial x} d x d t- \\
& -\int_{t_{0}}^{t_{1}} \int_{x_{0}}^{x_{1}}\left[\int_{t_{1}}^{t_{2}} \int_{x}^{x_{1}} p_{2}^{\prime}(\tau, s) C_{2}(\tau, s) \frac{\partial B(s)}{\partial s} d s d \tau\right] \frac{\partial^{2} \Delta z_{1}(t, x)}{\partial t \partial x} d x d t-
\end{aligned}
$$




$$
\begin{gathered}
-\int_{t_{0}}^{t_{1}} \int_{x_{0}}^{x_{1}}\left[\int_{t_{1}}^{t_{2}} p_{2}^{\prime}(\tau, x) C_{2}(\tau, x) B(x) d \tau\right] \frac{\partial^{2} \Delta z_{1}(t, x)}{\partial t \partial x} d x d t- \\
-\int_{t_{1}}^{t_{2}} \int_{x_{0}}^{x_{1}}\left[\int_{t}^{t_{2}} p_{2}^{\prime}(\tau, x) C_{2}(\tau, x) d \tau\right] \frac{\partial^{2} \Delta z_{2}(t, x)}{\partial t \partial x} d x d t-\int_{t_{1}}^{t_{2}} \int_{x_{0}}^{x_{1}} \Delta_{\bar{u}_{2}} M\left[t, x, p_{2}\right] d x d t .
\end{gathered}
$$

Assume that $p_{i}(t, x), i=\overline{1, k}$ are the solution to the linear Volterra integral equations

$$
\begin{gathered}
p_{1}(t, x)=-\sum_{i=1}^{k} \alpha_{i}(t, x) \frac{\partial \varphi_{1}{ }^{\prime}\left(z_{1}\left(T_{1}, X_{1}\right), \ldots, z_{1}\left(T_{k}, X_{k}\right)\right)}{\partial z_{1}}- \\
-\sum_{i=1}^{k} \gamma_{i}(x) B\left(X_{i}\right) \frac{\partial \varphi_{2}{ }^{\prime}\left(z_{2}\left(\theta_{1}, X_{1}\right), \ldots, z_{2}\left(\theta_{k}, X_{k}\right)\right)}{\partial z_{2}}+\int_{t}^{t_{1}} \int_{x}^{x_{1}} p_{1}^{\prime}(\tau, s) A_{1}(\tau, s) d s d \tau+ \\
+\int_{x}^{x_{1}} p_{1}^{\prime}(t, s) B_{1}(t, s) d s+\int_{t}^{t_{1}} p_{1}^{\prime}(\tau, x) C_{1}(\tau, x) d \tau+\int_{t_{1}} \int_{x}^{t_{2}} p_{2}^{\prime}(\tau, s) A_{2}(\tau, s) B(s) d s d \tau+ \\
p_{2}(t, x)=-\sum_{i=1}^{t_{2}} \beta_{i}(t, x) \frac{\partial \varphi_{2}{ }^{\prime}\left(z_{2}\left(\theta_{1}, X_{1}\right), \ldots, z_{2}\left(\theta_{k}, X_{k}\right)\right)}{\partial z_{1}}+\int_{t}^{t_{2}} \int_{x}^{t_{1}} p_{2}^{\prime}(\tau, s) A_{2}(\tau, s) B(s) d s d \tau \\
+\int_{x}^{t_{1}} p_{2}^{\prime}(t, s) B_{2}(t, s) d s+\int_{t}^{t_{2}} p_{2}^{\prime}(\tau, x) C_{2}(\tau, x) d \tau .
\end{gathered}
$$

Then the formula for increment (32) takes the form

$$
\begin{aligned}
\Delta S\left(u_{1}, u_{2}\right)= & -\int_{t_{0}}^{t_{1}} \int_{x_{0}}^{x_{1}} \Delta_{\bar{u}_{1}} M\left[t, x, p_{1}\right] d x d t-\int_{t_{1}}^{t_{2}} \int_{x_{0}}^{x_{1}} \Delta_{\bar{u}_{2}} M\left[t, x, p_{2}\right] d x d t+ \\
& +o_{1}\left(\sum_{i=1}^{k}\left\|\Delta z_{1}\left(T_{i}, X_{i}\right)\right\|\right)+o_{2}\left(\sum_{i=1}^{k}\left\|\Delta z_{2}\left(\theta_{i}, X_{i}\right)\right\|\right) .
\end{aligned}
$$

By virtue of the convexity of the functions $\varphi_{i}(),. i=1,2$, it follows from (33) that

$$
\Delta S\left(u_{1}, u_{2}\right) \geq-\int_{t_{0}}^{t_{1}} \int_{x_{0}}^{x_{1}} \Delta_{\bar{u}_{1}} M\left[t, x, p_{1}\right] d x d t-\int_{t_{1}}^{t_{2}} \int_{x_{0}}^{x_{1}} \Delta_{\bar{u}_{2}} M\left[t, x, p_{2}\right] d x d t .
$$

Using inequality (34), we prove

Theorem 2. For the optimality of the admissible control $\left(u_{1}(t, x), u_{2}(t, x)\right)$ in problem (1)(5), (28) it is necessary and sufficient that the inequalities

$$
\int_{t_{0}}^{t_{1}} \int_{x_{0}}^{x_{1}} \Delta_{\bar{u}_{1}(t, x)} M\left[t, x, p_{1}\right] d x d t \leq 0
$$




$$
\int_{t_{1}}^{t_{2}} \int_{x_{0}}^{x_{1}} \Delta_{\bar{u}_{2}(t, x)} M\left[t, x, p_{2}\right] d x d t \leq 0,
$$

hold for all $v_{i}(t, x) \in U_{i},(t, x) \in D_{i}, i=1,2$, respectively.

\section{Conclusion}

Using a version of the method of increments, a formula for the increment of the multi-point quality functional has been constructed. The constructed increment formula has made it possible to prove a necessary and sufficient optimality condition of the Pontryagin maximum principle type in the problem under consideration.

\section{References}

[1] А.И. Егоров, Оптимальном управлении процессами в некоторых системах с распределенными системами, Автоматика и телемеханика. No.5 (1964) pp.613-623. [In Russian: A.I. Yegorov, Optimal control of processes in some systems with distributed systems, Avtomatika i telemekhanika].

[2] А.И. Егоров, Оптимальные процессы в системах с распределенными параметрами и некоторые задачи теории инвариантности, Изв. АН СССР, Сер. матем. 29 No.5 (1965) pp.1205-1260. [In Russian: A.I. Yegorov, Optimal processes in systems with distributed parameters and some problems in the theory of invariance, Izv. AN SSSR, Ser. matem].

[3] В.И. Сумин, Функциональные вольтерровы уравнения в теории оптимального управления распределенными системами, Часть I, Н/Н, Изд-во ННГУ, (1992) 110 р. [In Russian: V.I. Sumin, Functional Volterra equations in the theory of optimal control of distributed systems, Part I, N/N, Izd-vo NNGU].

[4] С.С. Ахиев, К.Т. Ахмедов, Необходимые условия оптимальности для некоторых задач теории оптимального управления, Докл. АН Азерб. ССР. No.5 (1972) pp. 12-16. [In Russian: S.S. Akhiev, K.T. Ahmadov, Necessary optimality conditions for some problems in optimal control theory, Dokl. AN Azerb. SSR].

[5] О.В. Васильев, В.А. Срочко, В.А. Терлецкий, Методы оптимизации и их приложения, Часть I, Новосибирск, Наука, (1990) 190 p. [In Russian: O.V. Vasilyev, V.A. Srochko, V.A. Terletsky, Optimization methods and their applications, Part I, Novosibirsk, Nauka].

[6] Т.К. Меликов, Особые в классическом смысле управления в системах Гурса-Дарбу, Баку, Изд-во «ЭЛМ», (2003) 96 p. [In Russian: T.K. Melikov, Classically singular controls in the Gursa-Darbu systems, Baku, ELM].

[7] К.Б. Мансимов, М.Дж. Марданов, Качественная теория оптимального управления системами Гурса-Дарбу, Баку, ЭЛМ, (2010) 363 р. [In Russian: К.В. Mansimov, M.J. Mardanov, Qualitative theory of optimal control of Goursat-Darboux systems, Baku, ELM].

[8] К.Б. Мансимов, В.А. Сулейманова, Необходимые условия оптимальности в одной граничной задаче оптимального управления системами Гурса-Дарбу, Вест. Томск. Гос. Ун-та., матем. и мех.,No.9 (2017) pp. 26-42. [In Russian: K.B. Mansimov, V.A. Suleymanova, Necessary optimality conditions in a boundary value problem of optimal control of Goursat-Darboux systems, Vest. Tomsk. Gos. Un-ta., matem. i mekh].

[9] В.В. Величенко, Оптимальное управление составными системами, ДАН СССР. 176 No.4 (1967) pp.154-156. [In Russian: V.V. Velichenko, Optimal control of compound systems, DAN SSSR].

[10] С.Б. Кириченко, Оптимальное управление системами с промежуточными фазовыми ограничениями, Кибернетика и системный анализ. No.4 (1994) pp.104-111. [In Russian: S. B. Kirichenko, Optimal control of systems with intermediate phase constraints, Kibernetika i sistemnyy analiz].

[11] Г.К. Захаров, Оптимизация ступенчатых систем с управляемыми условиями перехода, Автоматика и телемеханика. No.1 (1993) pp.32-36. [In Russian: G.K. Zakharov, Optimization of step systems with controlled transition conditions, Avtomatika i telemekhanika].

[12] В.Н. Розова, Оптимальное управление ступенчатыми системами с неинтегральными функциональном, Вестник РУДН, сер. прикл. матем. и компютерная математика. 1 No.1 (2002) pp.131-136. [In Russian: V.N. Rozova, Optimal control with step systems with non-integral functional, Vestnik Rossiyskogo univ. druzhby narodov, Ser. prikl. i komp. Matematika].

[13] Р.Р. Исмайлов, К.Б. Мансимов, Об условиях оптимальности в одной ступенчатой задаче управления, Журн. выч. мат. и мат. физики. No.10 (2006) pp.1758-1770. [In Russian: K.B. Mansimov, R.R. Ismayilov, On optimality conditions in one step control problem, Zhurnal vychisl. matem. i matem. fiziki]. 